\title{
Morphological variants of nerve to gastrocnemius muscle, an anatomical guide to perform surgical dissection
}

\author{
V. Prathapamchandra ${ }^{1}$, L. V. Prabhu², M. M. Pai ${ }^{2}$ B.V. Murlimanju², R. Vadgaonkar² \\ 1 Senior Resident, Department of Anatomy, Jawaharlal Institute of Postgraduate Medical Education and \\ Research, Dhanvantri Nagar, Puducherry, India \\ 2 Department of Anatomy, Kasturba Medical College, Mangalore-575004, Manipal Academy of Higher \\ Education, Manipal, Karnataka, India.
}

\section{CORRESPONDING AUTHOR: \\ Latha V. Prabhu, Department \\ of Anatomy, Kasturba Medical \\ College, Manipal Academy of \\ Higher Education, Mangalore - \\ 575004, India. Telephone No- \\ +918242211746, \\ Telefax No- +91 8242421283, \\ E-Mail: \\ latha.prabhu@manipal.edu}

DOI:

10.32098/mltj.01.2019.12

\begin{abstract}
SUMMARY
Background. The precise knowledge about distribution of nerves supplying the gastrocnemius muscle is clinically important. The present study aimed to observe the morphological variations in the nerve supply of gastrocnemius in a sample South Indian population. Methods. The study included 38 formalin fixed adult cadaveric lower limbs. Variation in the number and branching pattern of the nerve to gastrocnemius muscle was studied. An imaginary line connecting the medial and lateral epicondyles of the femur, was considered as the reference line to study the topographical origin of the nerves. Results. It was observed that tibial nerve was giving one, two or three branches to the medial head of gastrocnemius muscle in $47.4 \%, 44.7 \%$ and $7.9 \%$ cases, respectively. The lateral head of gastrocnemius was receiving single, double or triple branch from tibia nerve in $60.5 \%, 34.2 \%$ and $5 \%$ cases, respectively. The nerve that innervated lateral head of gastrocnemius was also supplying soleus in $81.6 \%$ cases. The nerve to medial head was found at a higher level than the nerve to lateral head in $27(71.1 \%)$ cases, whereas in $11(28.9 \%)$ cases both were originating at the same level. In $76.3 \%$ cases, the nerve to MG was originating above the reference line and in $23.7 \%$ cases, below it. The nerve to LG was diverged below the reference line in $84.2 \%$ cases, at the reference line in $7.9 \%$ cases and above it in $7.9 \%$ cases. Conclusion. We report variations in the number and topography of nerves supplying the gastrocnemius muscle, that are enlightening to the operating plastic surgeon during the procedures like triceps surae nerve block and calf reduction. This data can be used as an anatomical guide to perform the surgical dissection.
\end{abstract}

\section{KEY WORDS}

gastrocnemius muscle; plastic surgery; soleus muscle; tibial nerve; Reconstructive Surgical Procedures

\section{BACKGROUND}

The gastrocnemius is one among the several muscles, which is used as graft in the flap surgeries. It serves as the best graft material to cover larger soft tissue defects of knee joint and lower leg (1). The gastrocnemius flap is considered as safest, for procedures like total knee arthroplasty (2) and can be used to cover defects in post-traumatic injuries. Gastrocnemius along with soleus outlines the calf contour. In this modern era, plump and muscular calves, so called "radish like legs", have become an aesthetic problem among the young Asian females. Fortunately, this can be achieved by calf reduction techniques which include liposuction, total or partial resection of the gastrocnemius muscle $(3,4)$, selective neurotomy $(5)$, Botulinum toxin injection (6) and radiofrequency volume reduction (7). However, these procedures are associated with postoperative complications like sensory loss, gait disturbances and compensatory muscular hypertrophy (8).

In certain upper motor neuron syndrome, which is associated with equinus deformity of the leg, gastrocnemius is involved, and in such situation spasticity of this muscle can be treated by procedures like neurolysis, motor point blocks by using phenol and alcohol or botulinum toxin (9). All these procedures require precise knowledge of distribution of the nerves to gastrocnemius muscle and morphological knowledge about these nerves help the operat- 
ing surgeon in preventing post-operative complications associated with these procedures (10). Since there are no anatomical reports available in literature, about variations in the innervation of gastrocnemius muscle, this anatomical study was undertaken. The aim of this investigation was to observe the morphological variants of the nerve to gastrocnemius muscle in a sample South Indian population.

\section{MATERIALS AND METHODS}

We included 38 embalmed adult human cadaveric lower limbs, obtained from the Department of Anatomy of our institution. The sample size calculation was based on a similar study (12), available in the literature. The details of age and gender were not available, so they are not considered. The lower limbs with surgical scars in the posterior compartment of leg and those with congenital malformations were excluded. This investigation has been approved by the institutional ethics committee, with respect to the human and animal rights and meets the scientific and ethical standards of the journal as discussed by Padulo et al. (11). The desired statistical power was set at $90 \%$, at a significance level of $5 \%$.

The dissection of the area to be studied was carried out as mentioned in Cunningham's Manual of Practical Anatomy (13). The popliteal fossa was explored and the branches of tibial nerve supplying the medial (MG) and lateral (LG) heads of gastrocnemius were identified. These branches were then traced till their entrance into the muscle by retracting MG and LG. An imaginary line connecting the medial and lateral epicondyles of the femur was considered as the reference line (RL).

The branches to MG and LG were studied with respect to their number and distance of diverging point of these nerve branches from the RL was estimated by using the digital Vernier caliper (Aerospace). The morphological variations of the branching patterns of nerves were observed and classified, based on their level of diversion from the tibial nerve (figure 1) as previously done by Hwang et al. (10).

The distal attachment of gastrocnemius to Achilles tendon was considered as the musculotendinous junction and the sizes of MG and LG were noted at this level. The sizes of the heads were compared with the branching patterns of nerve to the heads. The data analysis was performed by using SPSS version 13.0 (SPSS Inc., Chicago, IL, USA). Chi-square test was applied to evaluate the association between branching pattern of nerves and size of the heads at the musculotendinous junction, and p-value less than 0.05 was considered as statistically significant, as previously described (14).

\section{RESULTS}

It was observed that the nerve to MG and LG was from tibial nerve in all cases $(100 \%)$. In $81.6 \%$ cases, the nerve to LG gave a branch to soleus muscle.

\section{Nerve to medial head of gastrocnemius (MG)}

The nerve to MG diverged from tibial nerve as a single trunk, which further divided into 1-3 branches (figure 2a,

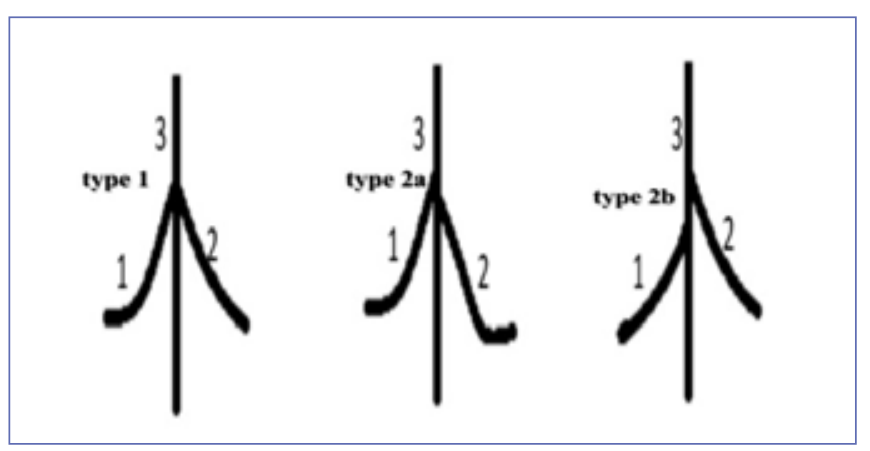

Figure 1 - Schematic diagram showing the branching pattern of nerves to MG and LG according to Hwang et al. (10) (MG, medial head of gastrocnemius; LG, lateral head of gastrocnemius; type 1, both nerves originating at same level; type 2a, nerve to $M G$ arising at a higher level than nerve to $L G$; type $2 b$, nerve to $M G$ arising at a lower level than nerve to $L G ; 1$, nerve to MG; 2 - nerve to LG; 3 , tibial nerve).

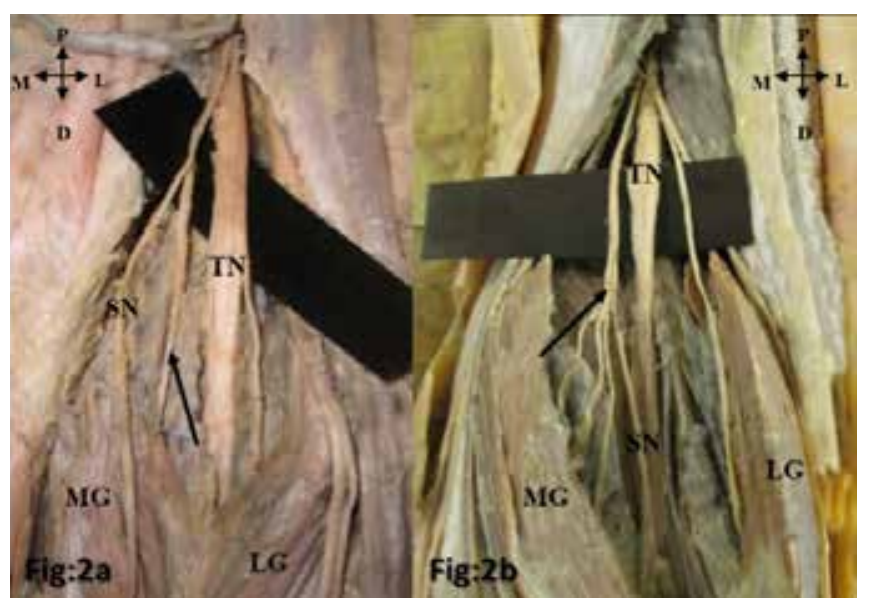

Figure 2a - Cadaveric leg showing nerve to $M G$ originating as single branch from the tibial nerve (arrow);

Figure $\mathbf{2} \mathbf{b}$ - Cadaveric leg showing nerve to $M G$ originating as three branches from the tibial nerve (arrow). (MG, medial head of gastrocnemius; LG, lateral head of gastrocnemius; $T N$, tibial nerve; $S N$, sural nerve; $M$, medial; $L$, lateral; $P$, proximal; D, distal) 
2b). This was one branch, two branch, and three branch in $47.4 \%, 44.7 \%$ and $7.9 \%$ cases respectively (table I). In 29 cases $(76.3 \%)$, the nerve to MG aroused above the RL and in the remaining 9 cases $(23.7 \%)$, it diverged below the RL (table II). The chi-square test analysis showed that the difference was not statistically significant $(\mathrm{p}=0.841)$. The point of origin varied from $2.8 \mathrm{~cm}$ above to $2.8 \mathrm{~cm}$ below the RL.

\section{Nerve to lateral head of gastrocnemius (LG)}

The nerve to LG diverged as a single trunk from the tibial nerve, which further divided into 1-3 branches (figure 3 a, $3 \mathrm{~b})$. It had single branch, two branch and three branch, in $60.5 \%, 34.2 \%$ and $5.3 \%$ cases respectively (table I). In 32 cases $(84.2 \%)$ the nerve diverged below the RL, in $3(7.9 \%)$ cases at the RL and in the remaining 3 cases $(7.9 \%)$ above the RL respectively (table II). The statistical comparison analysis by using Chi-square test was found to be statistically significant $(\mathrm{p}=0.041)$. The point of origin varied from 2.4 $\mathrm{cm}$ above to $3 \mathrm{~cm}$ below the RL.

\section{Morphological branching pattern of tibial nerve}

In the present study, the nerve to MG was arising at a level superior (type 2a branching pattern) than nerve to LG (figure $4 a)$ in $27(71.1 \%)$ cases. Both nerves were branching at the same level (figure $\mathbf{4 b}$ ) in $11(28.9 \%$ ) cases (type 1 branching pattern). It was also observed that type 1 branching pattern was associated with equal number of branches

Table I - Frequency of branches of nerve to MG and LG with respect to number.

\begin{tabular}{lll}
\hline Morphology of number & nerve to MG & nerve to LG \\
\hline one branch & $18(47.4 \%)$ & $23(60.5 \%)$ \\
\hline two branches & $17(44.7 \%)$ & $13(34.2 \%)$ \\
\hline three branches & $3(7.9 \%)$ & $2(5.3 \%)$ \\
\hline
\end{tabular}

MG, medial head of gastrocnemius ( $\mathrm{n}=38$ ); LG, lateral head of gastrocnemius $(\mathrm{n}=38)$.

Table II - Topographical distribution of nerve MG and LG with respect to their level of origin.

\begin{tabular}{lll}
\hline Topography of branching & nerve to $\mathbf{M G}$ & nerve to LG \\
\hline above the RL & $29(76.3 \%)$ & $3(7.9 \%)$ \\
\hline at the RL & $0(0 \%)$ & $3(7.9 \%)$ \\
\hline below the RL & $9(23.7 \%)$ & $32(84.2 \%)$ \\
\hline $\begin{array}{l}\text { MG, medial head of gastrocnemius; } \\
\text { reference line. lateral head of gastrocnemius; RL, }\end{array}$
\end{tabular}

to both the heads. Whereas type 2a branching pattern was associated with a higher number of branches to MG.

\section{Relation between the branching pattern and size of gastrocnemius head}

It was observed that, the MG was larger than LG at the level of confluence of musculotendinous junction in $27(71.1 \%)$ lower limbs. Both the heads appeared almost equal in size
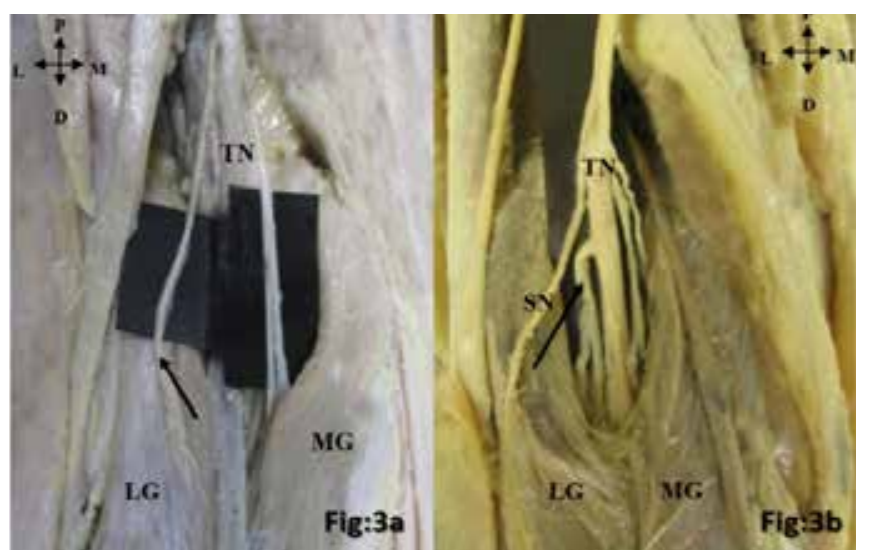

Figure $3 a$ - Cadaveric leg showing nerve to LG originating as single branch from the tibial nerve (arrow);

Figure $3 b$ - Cadaveric leg showing nerve to LG originating as two branches from the tibial nerve (arrow) (MG, medial head of gastrocnemius; LG, lateral head of gastrocnemius; TN, tibial nerve; SN, sural nerve; $M$, medial; L, lateral; $P$, proximal; $D$, distal).

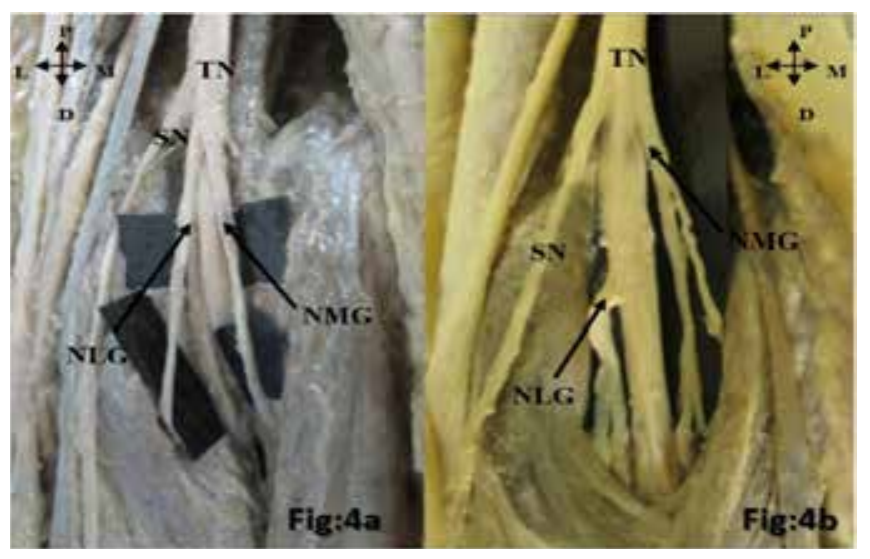

Figure 4a - Cadaveric leg showing nerve to medial (NMG) and lateral (NLG) heads of gastrocnemius arising from TN at the same level - type 1 (arrow), 28.9\% cases in this study; Figure 4b Cadaveric leg showing the NMG arising at a higher level than NLG from the TN - type 2 a (arrow), $71.1 \%$ of our cases (TN, tibial nerve; SN, sural nerve; $M$, medial; L, lateral; $P$, proximal; $D$, distal). 
in $11(28.9 \%)$ lower limbs. The Chi-square test showed a statistically significant association, between the branching pattern of nerves and the size of heads $(p=0.001)$. The statistical analysis exhibited that if the branching pattern was of type 2a, the size of MG was greater than the LG. The type 1 branching pattern was observed in cases where both heads of gastrocnemius were of equal size.

\section{DISCUSSION}

In an individual, the enlargement of calves may be due to excessive accumulation of subcutaneous fat or calf muscular hypertrophy (15). The management modalities include liposuction of the legs, if the subcutaneous fat is high (16). It is described that the subcutaneous fat at the medial part of the calf is soft, and is easier to suction than at the lateral region (17). However, for muscle hypertrophy, calf reduction procedures are performed (15). The management modalities include both conservative and surgical methods (18). Botox injection is a non-surgical method, which is quite expensive and requires repeated injections (6). The hypertrophy of calf and distorted legs are stressful to many female patients. The calf contouring procedures like liposuction, selective neurectomy and calf muscle resection are popular among Northeast Asians (19). The total resection of gastrocnemius muscle was first described by Lemperle and Exner (4). But the major disadvantages of this procedure were scarring, alteration in contour and shape of the legs. However, subtotal resection of gastrocnemius muscle was considered satisfactory, as this served the purpose of calf reduction without affecting the leg function and there was no hollow deformity at the popliteal region (20). It was reported that the postoperative compensatory hypertrophy is lesser in partial excision, when compared to total excision, as the function of muscle is still maintained (3).

After the procedure of neurectomy, the postoperative response in the form of volume reduction and subsequent change in the shape of the leg, are unpredictable (6). However, there are some advantages. The method of total resection of gastrocnemius muscle or neurectomy have the short recovery time and will leave minimal scar (10).

The clinical implications of the morphological knowledge of nerves supplying the gastrocnemius are high. Since there are no anatomical reports available about the morphology of nerve supply to the gastrocnemius muscle in Indian population, the present cadaveric cross sectional study was undertaken. We believe that the data of this study will enlighten the plastic surgeon about the nerve supply of gastrocnemius muscle. However, we did not examine the intramuscular branches of tibial nerve, but focused on the most accessible branches to the surgical dissection. We have observed that the nerve to MG and LG had more than one branch, in $52.6 \%$ and $39.5 \%$ cases, respectively. This finding is almost similar to the data published by Hawng et al. (10) and Liu et al. (21). However, the present study observed solitary branch of tibial nerve in $53.9 \%$ (MG and LG together) cases, which is lesser in frequency in comparison to the observations made by Sook Kim et al. (9) (90\%), and Abdel-Ghany (22) (100\%). This suggests that, it is not uncommon to see Indians having more than one branch of tibial nerve supplying the MG and LG.

Hawng et al. (9) observed the origin of nerve to soleus from the nerve to LG in $30 \%$ of cases. Abdel-Ghany (22) reported this morphological finding in $8.33 \%$ cases. However, we report a higher frequency of $81.6 \%$ cases. The present study showed that, in $76.3 \%$ of cases, the nerve to MG was originating from the tibial nerve above the RL. This is different from the findings of Yoo et al. (23), in which the nerve to $M G$ was proximal to the RL in only $0.2 \%$ of cases. The current study observed that the nerve to MG diverged at a higher level than the nerve to LG in $71.1 \%$ of cases (type 1). It was also observed that both the nerves originated at the same level in $28.9 \%$ of cases (type 2a). This is different from the previous observations made by Wang et al (24) who reported that the nerve to MG diverged higher than the nerve to LG in $95 \%$ of cases. Hwang et al. (10) observed type 1 branching pattern in $44 \%$ cases and type 2 a pattern in $48.6 \%$ cases, which also is differing from our study. The nerve to $\mathrm{MG}$ originating at a level inferior than nerve to $\mathrm{LG}$ (type 2b), was observed by Hwang et al. (10) in 7\% of cases. Such a pattern was not spotted in the sample limbs of the present study.

An interesting observation was that the size of LG was either less than or equal to that of MG. This may be the reason for lower branching of the nerve to LG, among our specimens. Also in our specimens, the greater contribution to the formation of musculotendinous junction was by $\mathrm{MG}$ ( $71.1 \%$ of cases). Nitya et al. (25) observed a higher incidence of $93.3 \%$ in their specimens. We witnessed that type 1 branching pattern was associated with equal size of $M G$ and LG, and also to equal number of branches of nerves, whereas type 2 a pattern was associated with larger size of MG and higher number of branches.

The present investigation observed that MG and LG can have more than one branch arising from the tibial nerve. In this context, we opine that, unless all the branches of tibial nerve are excised during the procedure of neurectomy, the hypertrophy of the calf muscles can recur. We suggest that the nerves to MG and LG can be easily located by placing a nerve stimulator, from $2.8 \mathrm{~cm}$ above to $3 \mathrm{~cm}$ below the $\mathrm{RL}$, which joins the two condyles of femur. However, unlike certain current literature, we didn't observe the microscop- 
ic/histological structure of gastrocnemius and its nerve, which is a limitation of this study.

\section{CONCLUSION}

The findings of this study suggest that the branching pattern of tibial nerve supplying the gastrocnemius muscle is unique for Indian population. The findings didn't match with similar studies from other geographical location. We suggest that both the geographical location and ancestry should be taken into consideration. This study has shown varied and additional information about the nerve supply of MG and LG. Since most of the data available in the literature are from the

\section{REFERENCES}

1. Schmidt I. The role of gastrocnemius muscle flap for reconstruction of large soft tissue defects after infected total knee arthroplasty. Int J Case Rep Images 2017; 8:7-10.

2. Castorina S, Guglielmino C, Castrogiovanni P, et al. Clinical evidence of traditional vs fast track recovery methodologies after total arthroplasty for osteoarthritic knee treatment. A retrospective observational study. Muscles Ligaments Tendons J 2017; 7:504-513.

3. Kim IG, Hwang SH, Lew JM, Lee HY. Endoscope-assisted calf reduction in Orientals. Plast Reconstr Surg 2000; 106:713-720.

4. Lemperle G, Exner K. The resection of gastrocnemius muscles in aesthetically disturbing calf hypertrophy. Plast Reconstr Surg 1998; 102:2230-2236.

5. Tsai FC, Mardini S, Fong TH, Kang JH, Chou CM. Selective neurectomy of the gastrocnemius and soleus muscles for calf hypertrophy: an anatomical study and 700 clinical cases. Plast Reconstr Surg 2008; 122:178-187.

6. Lee HJ, Lee DW, Park YH, Cha MK, Kim HS, Ha SJ. Botulinum toxin a for aesthetic contouring of enlarged medial gastrocnemius muscle. Dermatol Surg 2004; 30:867-871.

7. Park YJ, Jo YW, Bang SI, et al. Radiofrequency volume reduction of gastrocnemius muscle hypertrophy for cosmetic purposes. Aesthetic Plast Surg 2007; 31:53-61.

8. Park SS, Lew HS, Yang YM, et al. Analysis on complications of selective neurectomy. In: Proceedings of the 48th Congress of the Korean Society of Plastic and Reconstructive Surgeons. Pusan, Korea; May 13, 2000.

9. Sook Kim H, Hye Hwang J, Lee PK, et al. Localization of the motor nerve branches and motor points of the triceps surae muscles in Korean cadavers. Am J Phys Med Rehabil 2002; 81:765-769.

10. Hwang K, Kim YJ, Chung IH, Won HS, Tanaka S, Lee SI. Innervation of calf muscles in relation to calf reduction. Ann Plast Surg 2003; 50:517-522.

11. Padulo J, Oliva F, Frizziero A, Maffulli N. Muscles, Ligaments and Tendons Journal - Basic principles and recommendations in clinical and field Science Research: 2016 Update. Muscles Ligaments Tendons J 2016; 6:1-5.

12. Abdel-Ghany AGH, Ollo HA. Anatomical and Histological Study of Tendoachilles. Bull Alex Fac Med 2008; 44:833-839.
Western population, this study can be used as a database for the South Indian population. The morphological findings of the present investigation can be used as an anatomical guide for performing the surgical dissection. The data have got implications in plastic surgery and may enlighten the operating surgeon during surgical procedures like calf reduction and triceps surae nerve block.

\section{Conflict of interest}

We declare that we have no relationships, conditions and circumstances that present potential conflict of interest with this manuscript.

13. Cunningham DJ. The lower limb. In: Romanes GJ, ed. Cunningham's Manual of Practical Anatomy, Volume 1, Upper and Lower Limbs, 15th ed. Oxford; Oxford University Press 1986; 160-190.

14. Guglielmino C, Massimino P, Ioppolo F, Castorina S, Musumeci G, Di Giunta A. Single and dual incision technique for acute distal biceps rupture: clinical and functional outcomes. Muscles Ligaments Tendons J 2016; 6:453-460.

15. Kim SC, Kang MH, Ock JJ. Calf-contouring surgery of gastrocnemius hypertrophy: selective neurectomy of the sural nerve. Aesthetic Plast Surg 2008; 32:889-893.

16. Weniger FG, Calvert JW, Newton ED. Liposuction of the legs and ankles: a review of the literature. Plast Reconstr Surg 2004; 113:1771-1785.

17. Watanabe K. Circumferential liposuction of calves and ankles. Aesthetic Plast Surg 1990; 14:259.

18. Piekaar RSM, Zwitser EW, Hedeman Joosten PPA, Jansen JA. Painless swollen calf muscles of a 75 -year-old patient caused by bilateral venous malformations. World J Orthop 2017; 8:602-605.

19. Park SH, Kim JH, Lee JW, Jeong HS, Suh IS. Simultaneous use of selective neurectomy with liposuction for calf reduction in Asians. Aesthet Surg J 2018; 38:529-537.

20. Lee JT, Wang CH, Cheng LF, Lin CM, Huang CC, Chien SH. Subtotal resection of gastrocnemius muscles for hypertrophic muscular calves in Asians. Plast Reconstr Surg 2006; 118:14721483.

21. Liu DL, Li XI, Shan L, Li Q, Yuan JL, Yuan Q. The anatomic study and clinical observation of the neurectomy of the nerve to the medial gastrocnemius muscle for calf reduction. Zhonghua Zheng Xing Wai Ke Za Zhi 2007; 23:125-127.

22. Abdel-Ghany AGH. Anatomical study of the tibial nerve. Bull Alex Fac Med 2009; 45:759-770.

23. Yoo WK, Chung IH, Park CI. Anatomic motor point localization for the treatment of gastrocnemius muscle spasticity. Yonsei Med J 2002; 43:627-630.

24. Wang HB, Lin SQ, Xu DC, et al. Anatomic study of selective neurectomy of gastrocnemius muscle for calf reduction in Chinese. J Plast Reconstr Aesthet Surg 2013; 66:e162-e165.

25. Nitya J, Mariya. Morphological aspects of triceps surae - a cadaveric study. International Journal of Innovative Research and Development 2013; 2:372-376. 